\title{
IMPORTÂNCIA DO EXAME RADIOGRÁFICO NO DIAGNÓSTICO DE MEGAESÔFAGO EM CÃES
}

\author{
IMPORTANCE OF RADIOGRAPHIC EXAMINATION IN MEGAESOPHAGUS \\ DIAGNOSIS IN DOGS
}

\begin{abstract}
Álison Maciel dos Anjos (ANJOS, A. M.)
Discente do Curso Superior de Tecnologia em Radiologia, Faculdade Evangélica de Ceres, Ceres - GO, Brasil. maciel171@ outlook.com
\end{abstract}

João Paulo Gonçalves Ribeiro (RIBEIRO, J. P. G.)

Discente do Curso Superior de Tecnologia em Radiologia, Faculdade Evangélica de Ceres, Ceres-GO, Brasil. joaopaulogoncalvesribeiro@ outlook.com

\section{Danielle Muniz Pessoa Aires (AIRES, D. M. P.)}

Esp. Docente do Curso Superior de Tecnologia em Radiologia, Faculdade Evangélica de Ceres. Ceres - GO, Brasil. daniellempessoa@ hotmail.com

\section{Endereço para correspondência:}

Avenida Perimetral, Quadra 07, Lote 04, Setor São Francisco I, Santa Isabel - GO. Brasil. CEP: 76320-000.

\section{RESUMO}

INTRODUÇÃO: O megaesôfago é uma patologia comum em cães. Acontece pela ausência ou a diminuição demasiada dos plexos da parede do esôfago, ocasionando a expansão órgão, tornando-o frouxo e flácido e o peristaltismo incapaz de realizar movimentos normais. OBJETIVO: Discorrer sobre a importância do exame radiográfico para diagnosticar o megaesôfago, descrever a anatomia do órgão e ainda, conceituar a referida patologia. METODOLOGIA: O estudo foi realizado através de revisão de literatura do tipo narrativa, a partir de livros das bibliotecas física e virtual da Faculdade Evangélica de Ceres e artigos científicos de base de dados virtuais como, Google Acadêmico e Scielo. DISCUSSÃO: O megaesôfago pode ser classificado em congênito, idiopático adquirido, por problemas no arco aórtico direito e secundário. Além do exame clínico, para confirmar o diagnóstico em ocorrência de megaesôfago os exames radiográficos simples e contrastados são primordiais. Neste sentido, o raio-X vem sendo utilizado por ser o mais acessível para as clínicas e proprietários de cães. CONCLUSÃO: O exame radiográfico simples e em especial o contrastado é de grande valia para diagnosticar o megaesôfago em cães com precisão; auxilia tanto no diagnóstico quanto no tratamento da patologia, proporcionando melhor qualidade de vida ao animal.

Palavras chaves: Cão. Esôfago. Exame radiográfico. Megaesôfago. 


\begin{abstract}
INTRODUCTION: Megaesophagus is a usual pathology in dogs. Which occurs by the absence or too much reduction of the plexus of the esophagus wall, causing organ expansion, making it loose and flaccid, and peristalsis unable to perform normal movements. OBJECTIVE: To discuss the importance of the radiographic examination to diagnose megaesophagus, to describe the anatomy of the organ and to conceptualize the aforementioned pathology. METHODOLOGY: The study was carried out through literature review of the narrative type, from books of the physical and virtual libraries of the Evangelical Faculty of Ceres and scientific articles of virtual database such as Google Scholar and Scielo. DISCUSSION: Megaesophagus can be classified as congenital, acquired idiopathic, due to problems in the right and secondary aortic arch. In addition to the clinical examination, the simple and contrasted radiographic examinations are primordial to confirm the diagnosis in the occurrence of megaesophagus. In this sense, X-ray is being used for being the most accessible to clinics and dog owners. CONCLUSION: The simple radiographic examination and especially the contrasted one is of great value to diagnose megaesophagus in dogs with precision; assists both in the diagnosis and in the treatment of the pathology, providing a better quality of life to the animal.
\end{abstract}

Keywords: Dog. Esophagus. Megaesophagus. Radiographic examination.

\title{
1. INTRODUÇÃO
}

O esôfago tem a função de levar alimentos sólidos e líquidos até o estômago. As estruturas anatômicas que permitem esta função são representadas pelo músculo liso, controlado pelos mecanismos de reflexos, especialmente pelo nervo vago. Mas, isso também depende do alimento que passa pela faringe, que age estimulando o mecanismo de peristaltismo do esôfago, contraindo as partes superiores, relaxando as porções inferiores, fazendo com que o fluxo se mova em direção o estômago (BONFADA, 2005).

Quando a eliminação celular atinge níveis elevados, ocorre uma avançada desorganização de toda atividade motora e dilatação excessiva do órgão, ficando a movimentação esofágica restringida ou ausente, resultando em aglomeração ou retenção de alimentos e líquidos, podendo assim ocasionar o megaesôfago. Esta fisiopatologia acontece pela ausência ou a diminuição demasiada dos plexos da parede do esôfago, ocasionando um distúrbio do sistema esofágico na deglutição do alimento. A expansão do esôfago resulta em uma rigorosa desordem na motilidade, tornando esse órgão frouxo e flácido e o peristaltismo incapaz de ter seus movimentos normais (LONGSHORE, 2008; CELANO et al., 2018).

Essa alteração advém à medida que alguma parte do tubo esofágico restringe o seu ponto de extensão e em muitos casos são de origem hereditária, congênita ou de forma secundária. Quanto mais rápido se constatar esta patologia, a probabilidade de êxito do 
tratamento será maior. O diagnóstico é iniciado através de um exame físico detalhado da cavidade oral, região cervical e ausculta do tórax, com o intuito de sondar alterações orofaríngeas, dilatações ou "massas" na área do esôfago cervical, ou ainda, presença de pneumonia por aspiração, que pode complicar as doenças esofágicas $\mathrm{O}$ prognóstico depende da causa e do tempo dos acontecimentos dos sintomas (ANDRADE et al, 2007; SPILLMANN, 2007).

O reflexo da movimentação esofágica tem início quando os alimentos estimulam os neurônios sensoriais da mucosa esofágica, os comandos seguem para o centro de deglutição do tronco cerebral pelo nervo vago, que estimulam a contração dos músculos lisos e rugosos do esôfago. A lesão é passível de ocorrer em qualquer parte desse caminho, incluindo a junção de músculos e nervos, podendo resultar em alguma deficiência e distensão esofágica (TILLEY e SMITH, 2008).

Sabendo que o megaesôfago traz diversas complicações em animais e que existem várias formas de identificar esta patologia, o profissional, para fechar o diagnóstico, necessita de exames de imagem, sendo os mais acessíveis à clínica veterinária são as radiografias simples e contrastadas (WASHABAU, 2004).

Este trabalho teve como objetivo discorrer sobre a importância do exame radiográfico para o diagnóstico de megaesôfago em cães, descrevendo a anatomia do órgão e conceituando a referida patologia.

\section{METODOLOGIA}

O presente estudo foi realizado através de revisão de literatura do tipo narrativa, de caráter qualitativo. A pesquisa de material foi desenvolvida por meio de livros da biblioteca física e virtual da Faculdade Evangélica de Ceres, artigos científicos das bases de dados virtuais Google Acadêmico e Scielo, dissertações publicadas em repositórios, e ainda, trabalhos apresentados em eventos (congressos e seminários). Foram utilizados os buscadores "Cão", "Esôfago", "Exame radiográfico", "Megaesôfago".

Os critérios para a escolha das referências foram publicações de 2004 a 2018 nos idiomas português e inglês. A pesquisa obteve no levantamento de trabalhos 21 artigos científicos e destes, selecionados 13, pois traziam informações específicas ao tema. Os artigos descartados não traziam informações específicas, eram de base de dados não confiáveis ou por ser material muito antigo. Foram pesquisados ainda, 12 livros, todos incluídos no trabalho. 


\section{RESULTADOS E DISCUSSÃO}

Órgão de aspecto tubular, o esôfago é constituído por quatro camadas distintas de tecidos, que são chamados de adventícia, muscular, submucosa e camada mucosa. A camada muscular do esôfago do cão é inteiramente formada de músculo estriado. O epitélio da superfície é normalmente escamoso estratificado e o grau de queratinização é referente à dieta rotineira de uma espécie. Isto é notável quando comparado o epitélio esofágico do cão com o de um caprino, que consome alimentação mais dura (FEITOSA, 2014).

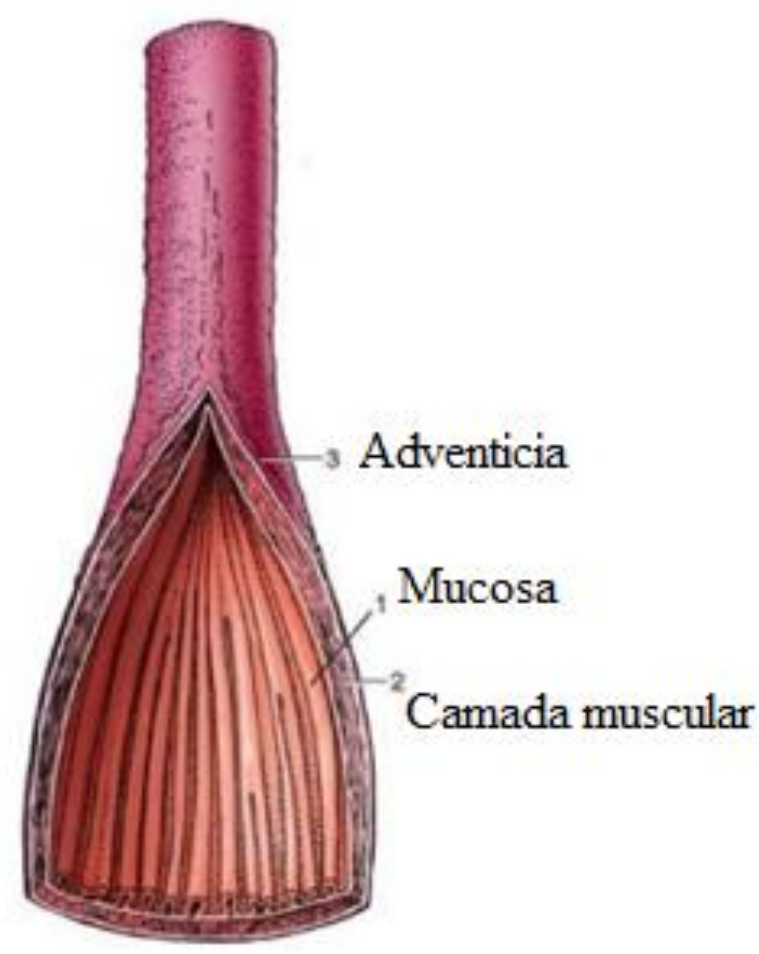

FIGURA 1 - Representação esquemática da estrutura esofágica. 2004)

FONTE: (DYCE et al.,

O esôfago é inervado pelos nervos simpáticos e nervos vagos e também se inclui os ramos laríngeos. O músculo estriado origina no mesoderma dos arcos faríngeos e é controlado pelos neurônios motores viscerais do vago, as partes de músculo liso são orientadas pelo sistema nervoso intrínseco e de forma indireta pelo sistema nervoso autônomo (DYCE et al., 2004).

O megaesôfago é uma fisiopatologia que resulta em uma expansão excessiva generalizada e difusa do órgão com a diminuição ou ausência do peristaltismo. Pode decorrer por anomalias congênitas ou por problemas adquiridos. As anomalias congênitas que resultam 
em quadros de megaesôfago podem ser resultados de anormalidades no anel vascular, divertículo esofágico e uma forma idiopática (TILLEY e SMITH, 2008).

É possível classificar o megaesôfago em congênito, idiopático adquirido ou por problemas no arco aórtico direito e secundário. No megaesôfago congênito os neurônios do centro da deglutição e os ramos sensoriais do cão que controla o peristaltismo demonstram falhas, ocasionando a patologia. Já no megaesôfago idiopático o cão apresenta dilatação esofágica em toda sua extensão, êmese, desidratação, perda de peso e fraqueza (SANTOS e ALESSI, 2016; TOMIO et al., 2016).

O megaesôfago sucedido pela persistência do arco aórtico direito se dá quando o esôfago apresenta dilatado na região torácica caudal aproximadamente da base do coração, apresentando estenose, esofagite focal e vestígios de alimentos nesta mesma região, deixando o esôfago flácido de maneira difusa e acentuada. Em todo caso, no megaesôfago secundário, o esôfago mostrará dilatado por toda sua extensão, apresentando hiperemia passiva e de maneira difusa acentuada, aspecto rígido e tonalidade cianótica acentuada (SANTOS, 2012).

Frequentemente no megaesôfago em canino ocorrem regurgitação e distúrbios de motilidade relacionados à redução do movimento de peristaltismo, o que leva a alterações no transporte de alimentos. O megaesôfago pode acometer a parte cervical ou torácica e os achados radiográficos dessa patologia podem incluir a dilatação do mesmo por gás e acumulo de líquidos ou alimentos, na faixa da traqueia, possibilitando a visualização do músculo longo do pescoço. Quando o esôfago é preenchido por gás, pode ser difícil fazer a sua diferenciação por causa da radiolucência que o circunda. Em relação às radiografias torácicas laterais, existem duas faixas radio-opacidade que diferencia das partes moles que direcionam da parte cranial para caudal em região do tórax (HAN e HURD, 2007).

Os cães com quadro de megaesôfago apresentam alguns sinais como, perda de peso, febre, vômito, crises de tosse, emagrecimento devido à dificuldade na deglutição, fraqueza, flacidez muscular, e o principal sinal, a regurgitação que ocorre logo após a ingestão do alimento. Para chegar a suspeita de megaesôfago inicia-se primeiramente a anamnese e exame físico, em seguida é solicitado o exame radiográfico, especialmente as radiografias contrastadas, podendo assim, chegar à conclusão definitiva do diagnóstico da patologia (ALVES, 2013; NELSON e COUTO, 2015).

Muitas patologias esofágicas podem ser detectadas por uma radiografia simples, já que a mistura de ar, líquidos e alimentos no esôfago podem delineá-lo, e assim, preenchê-lo parcialmente. Mas, para a visualização da estrutura com mais detalhes, é necessário o uso de contraste. Como a coluna vertebral fica sobreposta ao esôfago na posição ventro-dorsal é 
necessário que se realize uma radiografia látero-lateral e, se necessário, realiza-se a incidência dorsoventral. Na incidência ventrodorsal o cão é posicionado em decúbito dorsal, com os membros anteriores esticados, o esterno deve ficar sobreposto a coluna vertebral. Na láterolateral, o cão é colocado em decúbito lateral com as patas anteriores sentido cranialmente, o pescoço é estendido e o raio central é colocado no quinto espaço intercostal. Já na posição dorsoventral o cão em decúbito ventral, com a coluna sobreposta ao esterno, com as patas dianteiras esticadas (KEALY e MCALLISTER, 2005; BARROSO et al, 2005).

As radiografias simples não são capazes de concluir o diagnóstico, e por isso é sugerido que sejam realizadas radiografias contrastadas, através de meios de contraste baritados ou iodado. O tipo de contraste mais comum para o procedimento é à base de sulfato de bário, bastante utilizados em estudo de mucosa, já que se une a esta com mais facilidade. Quando há suspeita de perfuração, utiliza-se o contraste iodado, pois, com ele não ocorre a impregnação. A partir da mistura de alimento e contraste é possível visualizar dilatação intramural ou estenose de extensão. Quando da administração do contraste, é indicado que o procedimento seja realizado em plataformas elevadas com apoio para membros posteriores, pois este cuidado evita aspiração. É indicado ainda, a sedação do cão (SPILLMANN, 2007).

Em estudos contrastados do esôfago sem suspeita de perfuração utiliza-se o sulfato de bário, administrado via oral, na dosagem de 3 a $5 \mathrm{ml} / \mathrm{kg}$. Este meio de contaste é contraindicado em caso de ruptura esofágica, pois a impregnação em outros locais impedem a sua eliminação. As radiografias são iniciadas após toda porção de contraste ser ingerida. Situações em que o esôfago estiver muito dilatado é preciso uma quantidade maior de contraste para delinear a parede esofágica. Os exames radiográficos simples e contrastados são primordiais para confirmar o diagnóstico em ocorrências de megaesôfago, e para obter um diagnóstico exato de obstruções, corpos estranhos ou avaliar a motilidade é indispensável que seja realizado radiografias contrastadas (ETTINGER e FELDMAN, 2004; THRALL, 2014).

Segundo Fubini e Pease (2004) o exame preferencial para avaliar desordens ou lesão no esôfago em animais é a esofagografia. Estudos de Tomio (2016) citam que o diagnóstico da patologia pode ser realizado através de exames clínicos, confirmado através de esofagografia, corroborando com Silva Junior e colaboradores (2011), que, ao analisarem megaesôfago em caprinos, recomendaram a realização do exame contrastado para auxiliar efetivamente o diagnóstico da afecção. 
A figura 2 traz exemplo de radiografia simples evidenciando a presença de ar no esôfago, fato que reforça uma suspeita inicial de megaesôfago.

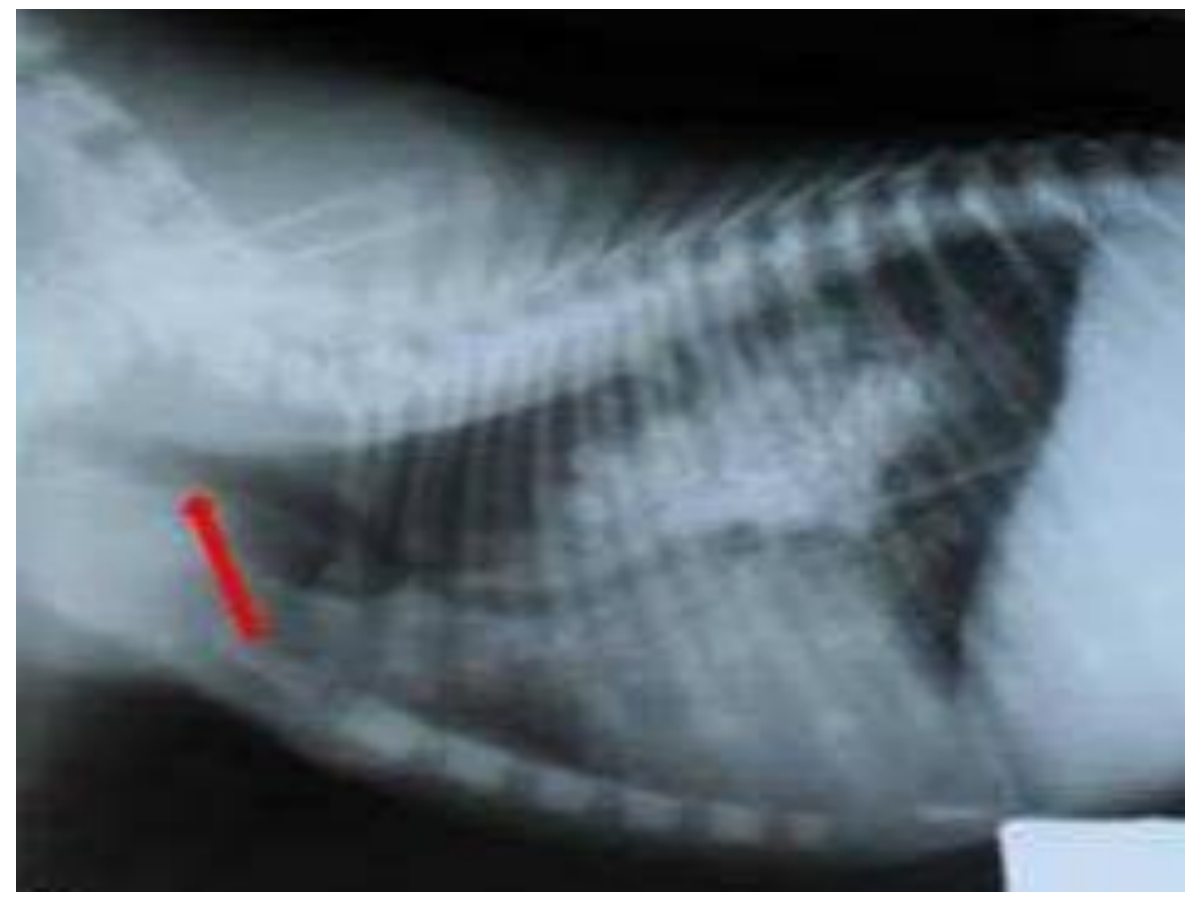

FIGURA 2 - Radiografia simples em incidência látero-lateral, evidenciando presença de ar em esôfago torácico. FONTE: (ALVES, 2013)

Já a figura 3 traz imagem, a partir de radiografia contrastada, que confirma o quadro da patologia garantindo um diagnóstico preciso.

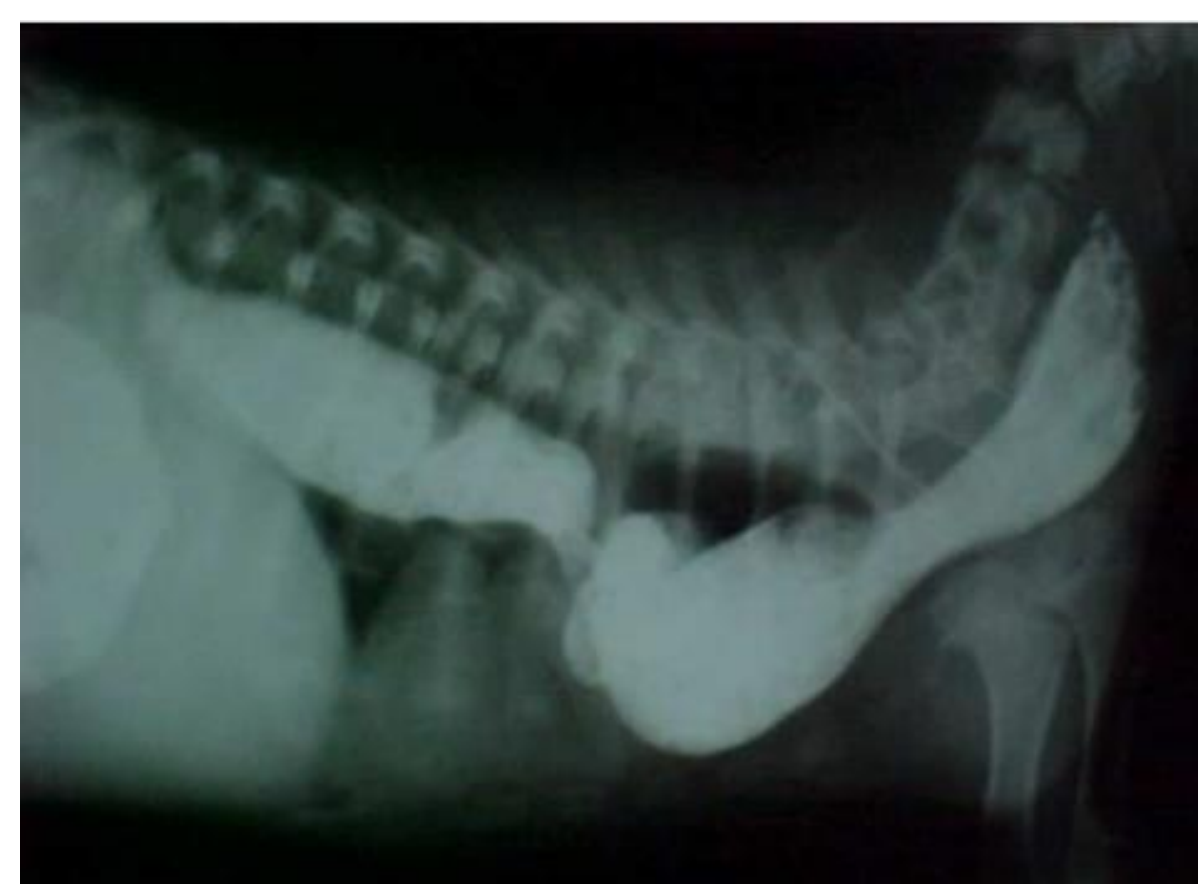

FIGURA 3 - Radiografia contrastada em incidência látero-lateral, evidenciando megaesôfago crânio caudal. FONTE: (OLIVEIRA et al, 2004) 
Após diagnóstico de megaesôfago, é possível optar pelo procedimento cirúrgico para correção da patologia, ou manter uso de medicação paliativa para amenizar o quadro. Neste sentido, o exame radiográfico contrastado da figura 4, evidência o esôfago já com redução considerável, 21 dias após tratamento cirúrgico (OLIVEIRA et al, 2004).

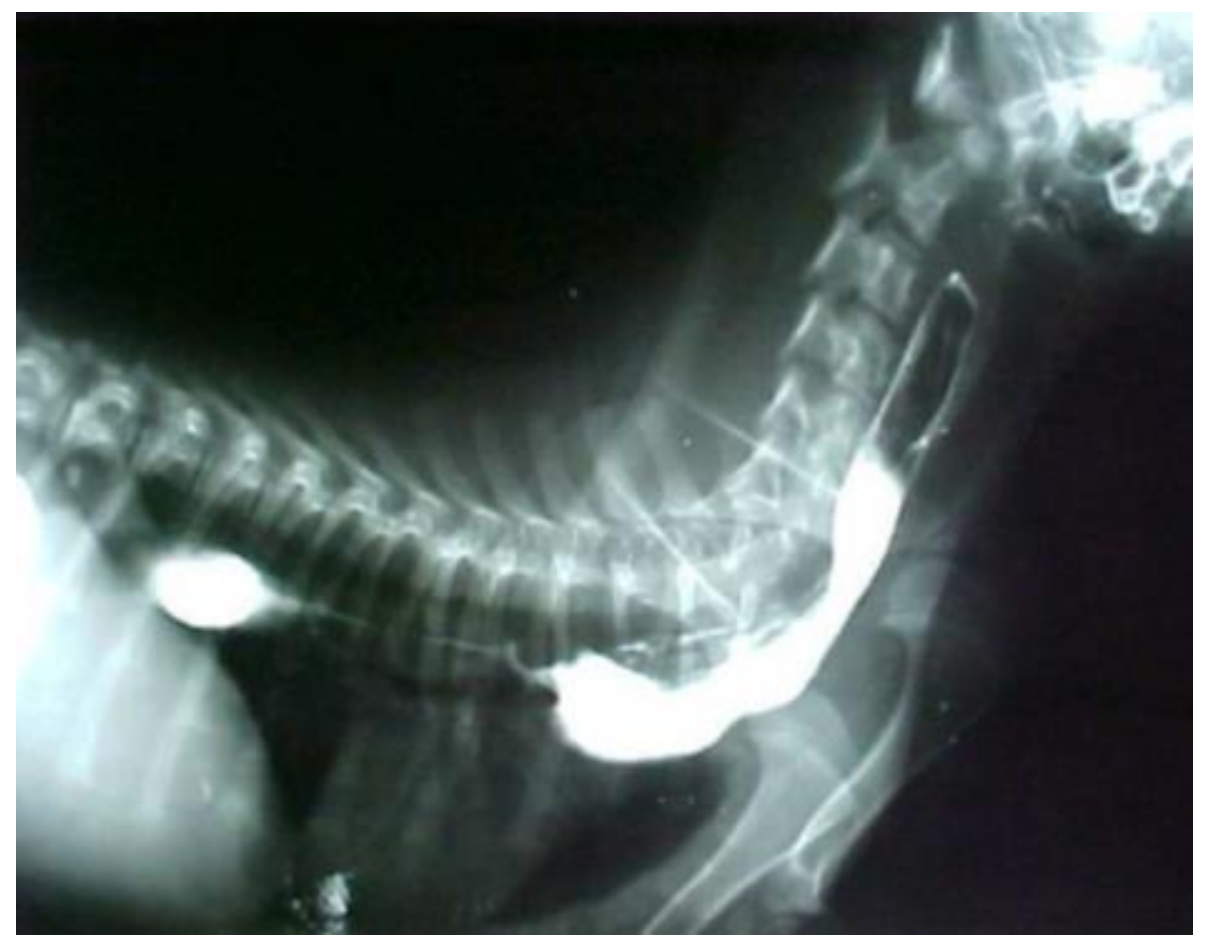

FIGURA 4 - Radiografia contrastada em incidência látero-lateral, evidenciando a redução do megaesôfago após 21 de cirurgia. FONTE: (OLIVEIRA et al, 2004)

Lopes (2008) reforça que a radiografia é o método mais acessível para a avaliação de patologias no esôfago de cães, sobretudo para diagnosticar o megaesôfago. É de fundamental importância que sejam realizadas radiografias simples e contrastadas do órgão para diagnóstico definitivo. Posteriormente, é possível fazer opção pelo tratamento que irá trazer melhora da qualidade de vida ao animal.

Em virtude da importância para o diagnóstico de patologias em animais, a radiologia veterinária vem cada dia mais se modernizado. Dos métodos de diagnóstico por imagem, o raios-X é o mais utilizado, pois é o mais acessível para as clínicas e para os proprietários dos animais, além de trazer imagens de qualidade e de baixo custo se comparado aos outros exames de imagem (ANDRADE, 2007). Ainda neste sentido, é importante ressaltar que é função do técnico ou tecnólogo em radiologia a realização do exame radiográfico, inclusive em medicina veterinária, conforme institui a Resolução do Conselho Nacional de Técnicos em Radiologia $\mathrm{n}^{\circ} 02$ de 10 de maio de 2005. 


\section{CONCLUSÃO}

O megaesôfago é uma fisiopatologia comum e recorrente em cães. A suspeita se dá a partir do exame clínico, mas a confirmação só é possível a partir de exames de diagnóstico por imagem. Para tanto, o exame radiográfico, em especial o contrastado, é o principal método para a obtenção do diagnóstico da patologia, auxiliando o profissional na escolha do tratamento, garantindo ao animal melhor qualidade de vida.

\section{REFERÊNCIAS}

ALVES, N. M.; SILVA, T.P.D.; PEREIRA, A.M.; ROSITO, J.; BORGES, M.M. Megaesôfago congênito em cão. PUBVET, Londrina, v. 7, n. 23, p. 2327-445, dezembro, 2013. Disponível em: <http://www.pubvet.com.br/uploads/ce04c52859ab25fb633f78b068a23bef.pdf >. Acesso em: 12.set.2018.

ANDRADE, S. A. F. Atuação do tecnólogo em radiologia na área da medicina veterinária. Revista UNILUS Ensino e Pesquisa, v. 4, n. 7, julho/dezembro.2007. Disponível em: <http://revista.unilus.edu.br/index.php/ruep/article/view/37>. Acesso em: 05.jul.2018.

ANDRADE, S. F.; BARILLI; R. M. N.; MELCHER; A.; SILVA, M. P. C.; MOTTA, Y. P.; BRINHOLI, R. B.; TOSTES, R. A.; SANCHES, O. Megaesôfago secundário à miastenia grave em uma cadela da raça Pastor Alemão. Redação de Revista Científica de América Latina y el Caribe, Espanha y Portugal, v. 28, n. 3, p. 477-82, julho - setembro. 2007. Disponível em: < https://www.redalyc.org/pdf/4457/445744085022.pdf>. Acesso em: 17.jul.2018.

BARROSO, R. M. V.; DE PAULA, T. M.; ÁVILA JR, R. Radiologia torácica. Revista REDVET., v. 6, n. 3, 2005.

Disponível em:

<http://www.imagingonline.com.br/waUpload/03050800105022014155428.pdf > Acesso em: 24.out.2018.

BONFADA, A. T. Cirurgia torácica vídeo assistida sem intubação seletiva com acesso modificado para sutura do esôfago caudal em cães. 2005. Dissertação (Mestrado em Medicina Veterinária) - Universidade Federal de Santa Maria, Santa Maria.

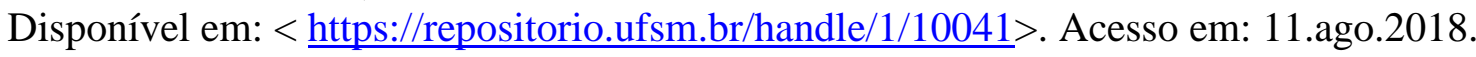


CELANO, R. M. G.; NETO, J.E.; BOTTONI, A.; GAGLIARDI, D. Avaliação nutricional pré-operatória dos pacientes com megaesôfago não-avançado. Revista do Colégio Brasileiro de Cirurgiões, v. 34, n. 3, p. 147-152, 2018.

Disponível em:

<http://www.scielo.br/scielo.php?pid=S010069912007000300003\&script=sci_abstract\&tlng= pt>. Acesso em: 12.jul.2018.

CONTER. CONSELHO NACIONAL DE TÉCNICOS EM RADIOLOGIA. Resolução CONTER No 2, 10 de Maio de 2005. Disponível em:

<http://conter.gov.br/uploads/legislativo/n. 022005.pdf>. Acessado em: 09.nov.2018

DYCE, K. M.; WENSING, C. J. G.; SACK, W. O. Tratado de anatomia veterinária. Brasil: Elsevier, 2004.

ETTINGER, J. S.; FELDMAN, C. E. Tratado de Medicina Interna Veterinária: Doença do cão e do gato, $5^{\text {a }}$ edição. Rio de Janeiro: Guanabara Koogan, 2004.

FEITOSA, F. L. F. Semiologia veterinária: a arte do diagnóstico, $3^{a}$ edição. São Paulo: Roca, 2014.

FUBINI, S. L.; PEASE. A. P. Esophagus surgery: Farm animal surgery. St. Lowis: Saunders, 2004.

HAN, C. M.; HURD, C. D. Diagnóstico por imagem para a prática veterinária. São Paulo: Roca, 2007.

KEALY, J. K.; MCALLISTER, H. Radiologia e Ultrassonografia do Cão e do Gato. Brasil: Elsevier, 2005.

LONGSHORE, R. C. Consulta Veterinária em 5 minutos: canina e felina, $3^{\text {a }}$ edição. São Paulo: Manole, 2008.

LOPES, L. R. Resultados imediatos e tardios do tratamento cirúrgico do megaesôfago não avançado pela técnica de HELLER-PINOTTI: Laparotomia versus Laparoscopia. 2008. Tese (Livre Docência na área de Moléstias do Aparelho Digestivo) - Faculdade de Ciências Médicas da Universidade Estadual de Campinas. Disponível em:

< http://repositorio.unicamp.br/handle/REPOSIP/313626>. Acesso em: 20.ago.2018.

NELSON, R.; COUTO, G. Medicina interna de pequenos animais. Brasil: Elsevier, 2015. 
OLIVEIRA, E. C.; GAIGA, L.L.; COLOMÉ, L.M.; STEDILE, R.; MELLO, F.P.S.; MARTINS, J.M.; FREIRE, C.D. Persistência do arco aórtico direito em um cão - Relato de caso. Revista da Faculdade de Zootecnia, Veterinária e Agronomia, v. 11, n. 1, 2004. Disponível em: $<$ http://revistaseletronicas.pucrs.br/ojs/index.php/fzva/article/view/2200/0>. Acesso em: 29.ago.2018.

SANTOS, R. L.; ALESSI, A. C. Patologia Veterinária, 2a Edição, São Paulo: Roca, 2016.

SANTOS, R. M. Avaliação anatomopatológica e causas de megaesôfago em cães. Revista Nucleus Animalium, v. 4, n. 2, novembro. 2012. Disponível em:

<http://nucleus.feituverava.com.br/index.php/animalium/article/view/799/1006>. Acesso em: 07.set.2018.

SILVA JÚNIOR, L. C.; ARRUDA, L. C. P.; SILVA, D. G. B.; SOARES, F. A. P.; BORBA NETO, A. V.; LEITE, J. E. B.; SOARES, P. C. Aspectos clínicos e radiográficos de caprino com megaesôfago. Arquivo Brasileiro de Medicina Veterinária e Zootecnia, Pernambuco, v. 63, n. 3, p. 761-764, 2011.

Disponível em: < http://www.scielo.br/scielo.php?pid=S0102-

$09352011000300033 \&$ script $=$ sci_arttext\&tlng $=$ pt $>$. Acesso em: 11.set.2018.

SPILLMANN, T. Esophageal diseases diagnostic and therapeutic approach. In: ANNUAL WSAVA CONGRESS. Sydney, 2007.

Disponível em:

<https://www.vin.com/apputil/content/defaultadv1.aspx?meta=Generic\&pId=11242\&id=3860 784>. Acesso em: 25.out.2018.

THRALL, D. Diagnóstico de radiologia veterinária. Brasil: Elsevier, 2014.

TILLEY, L. P.; SMITH, F. W. K. Consulta veterinária em 5 minutos, $2^{a}$ edição. Barueri: Manole, 2008.

TOMIO, J.; SANTOS, A. R. I.; FILHO, P. R. G.; TEICHMANN, C. E. Megaesôfago idiopático adquirido em cão. In: XXIV SEMINÁRIO DE INICIAÇÃO CIENTÍFICA, 2016, Rio Grande do Sul: Universidade Regional do Noroeste do Estado do Rio Grande do Sul, 2016. Disponível em: < file:///C:/Users/Cliente/Downloads/6348-1-27730-1-1020160912\%20(1).pdf>. Acesso em: 12.out.2018.

WASHBAU, R. J. Tratado de medicina interna veterinária: doença do cão e do gato, $5^{\text {a }}$ edição. Rio de Janeiro: Guanabara Koogan, 2004. 